\title{
Echocardiographic assessment of patent ductus arteriosus shunt flow pattern in premature infants
}

\author{
Bai-Horng Su, Toyoko Watanabe, Mitsumasa Shimizu, Masayoshi Yanagisawa
}

\begin{abstract}
Aims-To identify the patent ductus arteriosus (PDA) shunt flow pattern using Doppler echocardiography; and to assess whether it could be used to predict the development of clinically significant PDA. Methods-Premature infants weighing under $1500 \mathrm{~g}$, who required mechanical ventilation, and in whom daily echocardiography could be performed from day 1 until the ductus closed, and on day 7 to confirm closure, were studied. The PDA shunt flow was identified from four Doppler patterns, and the closed pattern of a closed duct was also presented. Clinically significant PDA was diagnosed when there was colour Doppler echocardiographic evidence of left to right ductal shunt associated with at least two of the following clinical signs: heart murmur (systolic or continuous); persistent tachycardia (heart rate $>160 / \mathrm{min}$ ); hyperactive precordial pulsation; bounding pulses; and radiographic evidence of cardiomegaly or pulmonary congestion.
\end{abstract}

Results-Of 68 infants enrolled into this study, clincally significant PDA developed in 31. The most recordable sequence of transition change of shunt flow pattern for clinically significant PDA was: pulmonary hypertension pattern, to growing pattern, to pulsatile pattern, to closing pattern, to closed pattern. And that for non-clinically significant PDA was: pulmonary hypertension pattern, to closing pattern, to closed pattern. The growing and the pulsatile patterns were mostly documented in infants with clinically significant PDA. The first documented growing pattern to predict clinically significant PDA gave a sensitivity of $64.5 \%$ and a specificity of $81.1 \%$; the first documented pulsatile pattern gave a sensitivity of $93.5 \%$ and a specificity of $100 \%$.

Conclusion-Doppler echocardiographic assessment of PDA shunt flow pattern during the first 4 days of life is useful for predicting the development of clinically significant PDA in premature infants. At that stage, the closing or closed Doppler pattern indicates that infants are not at risk of developing clinically significant PDA; the growing or pulsatile Doppler pattern indicates a continuing risk of developing clinically significant PDA. (Arch Dis Child 1997;77:F36-F40)

Keywords: Echocardiography; patent ductus arteriosus; Doppler flow pattern
Delayed closure of the ductus arteriosus is an important complication of neonatal intensive care. The introduction of surfactant replacement therapy to neonatal medicine has led to the clinical emergence of patent ductus arteriosus (PDA) earlier and more frequently in premature infants. ${ }^{1-3}$ Clinical diagnosis of a haemodynamically clinically significant PDA lacks accuracy. ${ }^{4-7}$ Therefore, echocardiographic evaluation has become essential for the clinical management of PDA and in assessing the magnitude of the duct. ${ }^{8-17}$

\section{Methods}

Premature infants weighing under $1500 \mathrm{~g}$ with respiratory distress that required mechanical ventilation for more than 24 hours were enrolled into this study. Echocardiographic assessment was carried out using a HewlettPackard 77020A or an Aloka SSD-2000 scanner with a $5 \mathrm{MHz}$ probe incorporating colour flow, pulsed and continuous wave Doppler ultrasonography. The first echocardiographic study confirmed cardiac normality and then serial echocardiographic studies were performed daily from day 1 until the ductus closed, and on day 7 to confirm ductal closure. Additional echocardiographic studies were performed every 6 to 12 hours if requested by the attending clinician, on the basis of physical signs, ventilatory requirements, and chest radiographic findings. The clinician was blinded to all the echocardiographic results except the colour Doppler flow pattern.

\section{PDA SHUNT FLOW PATTERN}

The ductus was imaged from the high left parasternal view using colour Doppler echocardiography. If PDA was present, the pulsed Doppler gate was carefully placed in the pulmonary end of the ductus to maintain the angle of insonation below 20 degrees and the flow pattern of the shunt was recorded (fig 1). Four Doppler patterns were identified, and the closed pattern of a closed duct was also presented (fig 2).

\section{Pulmonary hypertension pattem}

A bi-directional shunt was noted in the profile; a right to left shunt (downward away from the baseline) in early systole was followed by a small left to right shunt (upward away from the baseline) throughout the diastole. This pattern was seen in early postnatal life in the presence of high pulmonary vascular resistance.

Growing pattern

A bi-directional shunt still could be noted, but the right to left shunt decreased and a growing 
left to right shunt was seen in the profile. This pattern represents a growing left to right shunt through a large ductus accompanying a fall in pulmonary vascular resistance.

Table 1 Longitudinal change of Doppler PDA flow pattern and days of age in both clinically significant and non-significant PDA groups

\begin{tabular}{|c|c|c|c|c|c|c|c|c|c|c|}
\hline \multirow[b]{2}{*}{$P D A$} & \multicolumn{2}{|l|}{$P H$} & \multicolumn{2}{|c|}{ Growing } & \multicolumn{2}{|c|}{ Pulsatile } & \multicolumn{2}{|c|}{ Closing } & \multicolumn{2}{|c|}{ Closed } \\
\hline & $S$ & $N S$ & $S$ & $N S$ & $S$ & $N S$ & $S$ & $N S$ & $S$ & $N S$ \\
\hline Day 1 & 24 & 24 & 6 & 5 & 2 & & & 13 & & 1 \\
\hline Day 2 & & & 13 & 2 & 18 & & 5 & 16 & 1 & 6 \\
\hline Day 3 & & & 1 & & 8 & & 11 & 2 & 9 & 17 \\
\hline Day 4 & & & & & 1 & & 7 & & 9 & 10 \\
\hline Day 5 & & & & & & & 2 & & 5 & 3 \\
\hline Day 6 & & & & & & & 1 & & 3 & \\
\hline Day 7 & & & & & & & 1 & & 2 & \\
\hline
\end{tabular}

Values are number of infants with various Doppler PDA patterns documented on each day of age. Some infants had additional echocardiographic examination the same day. All the closing and closed patterns documented in the S-PDA group were the results of treatment. PDA: patent ductus arteriosus; S: significant PDA; NS: non-significant PDA; PH: pulmonary hypertension.

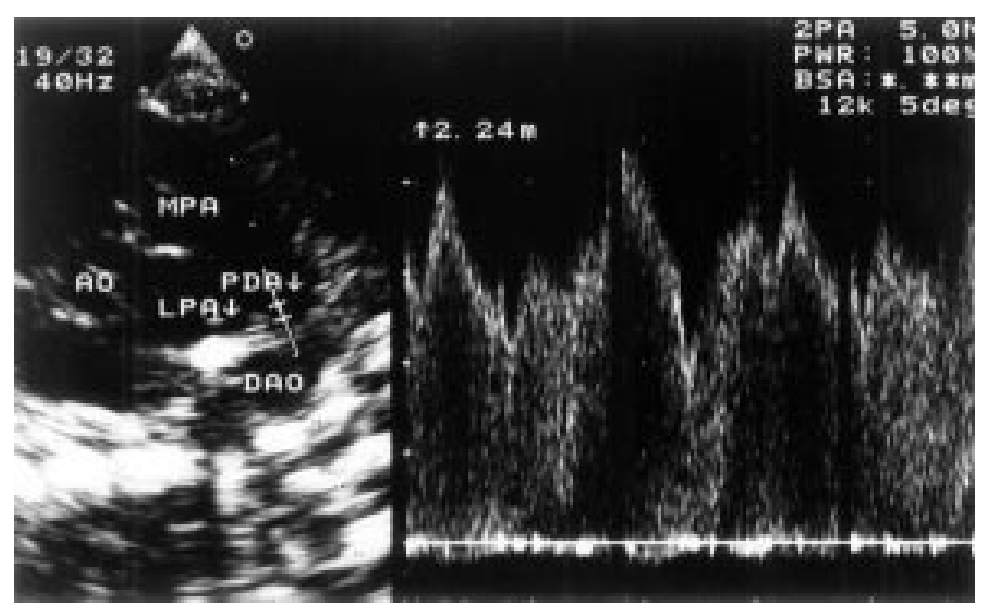

Figure 1 Left: high left parasternal view echocardiogram. The main pulmonary artery (MPA), patent ductus arteriosus (PDA), and descending aorta (DAO) can be seen in a contiuous arc. The origin of the left pulmonary artery (LPA) is also evident. Posterior to the DAO are the vertebral bodies. The pulsed Doppler gate is positioned at the pulmonary end of the PDA. Right: resultant Doppler profile of the the PDA shunt flow.
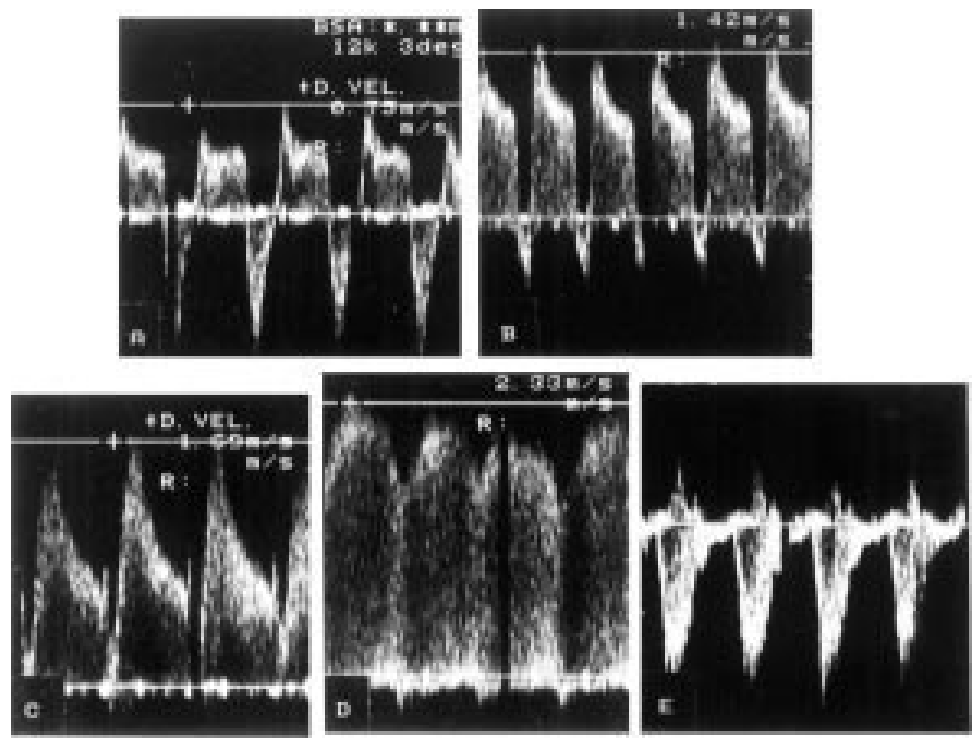

Figure 2 Four Doppler flow patterns and the contrasting closed pattern. A: pulmonary hypertension pattern; B: growing pattern; C: pulsatile pattern; D: closing pattern; E: closed pattern.
Pulsatile pattern

No right to left shunt was noted in the profile, and a much greater left to right shunt was shown by a pulsatile flow of peak velocity of about 1.5 metres/seconds.

\section{Closing pattern}

The prominent difference between this and the pulsatile pattern is that the closing pattern did not show the rhythmically pulsatile change, but rather a continuous left to right shunt with a peak flow velocity of about 2 milliseconds covering the whole cardiac cycle in the profile. According to the Bernoulli equation, this pattern implies that a shunt flows through a constrictive ductus to produce a high flow velocity.

\section{Closed pattern}

This pattern had to be sampled in the pulmonary artery, because the Doppler gate was placed in the pulmonary end of the ductus while it was closed, so true ductal sampling would have produced no Doppler signal at all. Thus the closed pattern is similar to the pulmonary artery flow pattern, and was taken to show the contrast with the PDA patterns.

Clinically significant PDA was diagnosed when there was colour Doppler echocardiographic evidence of left to right ductal shunt associated with at least two of the following clinical signs: heart murmur (systolic or continuous); persistent tachycardia (heart rate $>160 / \mathrm{min}$ ); hyperactive precordial pulsation; bounding pulses; and radiographic evidence of cardiomegaly or pulmonary congestion. The decision to treat an infant with indomethacin was made by the attending clinician who was blinded to all the echocardiographic results except the colour Doppler. Management of a clinically significant PDA in our unit includes fluid restriction and intravenous administration of indomethacin $(0.2 \mathrm{mg} / \mathrm{kg}$ for the initial dose, in infants less than 48 hours $0.1 \mathrm{mg} / \mathrm{kg}$, those over 48 hours $0.2 \mathrm{mg} / \mathrm{kg}$ every 24 hours for another two doses), if not contraindicated. ${ }^{18}$

The two tailed $t$ test and the $\chi^{2}$ test were used to test significance, a $\mathrm{P}$ value of $<0.05$ being regarded as significant. The sensitivity and specificity for the prediction of developing clinically significant PDA were calculated from the PDA Doppler pattern.

The study protocol was approved by the Research Institute of the Tokyo Metropolitan Tsukiji Maternity Hospital and the infants were studied after informed consent had been obtained from their parents.

\section{Results}

Sixty eight infants were enrolled into the study. Of these, 31 developed clinically significant PDA. Among these 31 infants, 29 were treated with indomethacin: in two infants the ductus reopened both at day 17 and closed again after the second course of indomethacin treatment and in another infant the ductus reopened at day 21 and closed again at day 28 with fluid restriction only. Two infants had contraindications to indomethacin treatment and were 
Table 2 Clinical status of PDA when various Dopller PDA patterns were initially documented during first 7 days of age

\begin{tabular}{lccccc}
\hline Clinical status of PDA & $P H(n=48)$ & Growing $(n=27)$ & Pulsatile $(n=29)$ & Closing $(n=58)$ & Closed $(n=66)$ \\
\hline NS-PDA group (n=37) & 24 & $7^{\star}$ & $0^{\star \star}$ & 31 & 37 \\
S-PDA group (n=31) & 24 & $20^{\star}$ & $29^{\star \star}$ & 27 & $29 \dagger$ \\
With S-PDA & 0 & 4 & 20 & 0 & 0 \\
Subsequent S-PDA & 24 & 16 & 9 & 0 & 0 \\
\hline
\end{tabular}

Values are number of infants with documented various Doppler PDA patterns.

All the closing and closed patterns in the S-PDA group were the result of treatment, and all non-clinically significant.

† Another two infants from the S-PDA group had a closed pattern that was first documented at 8 days of age.

${ }^{\star} \mathrm{P}=0.0001 ;{ }^{\star \star} \mathrm{P}<0.0001$.

Table 3 Percentage predictive power of first documented growing and pulsatile patterns for developing clinically significant PDA

\begin{tabular}{lllll}
\hline & Sensitivity & Specificity & Positive predictive value & Negative predictive value \\
\hline Growing pattern & $64.5(20 / 31)$ & $81.1(30 / 37)$ & $74.1(20 / 27)$ & $73.2(30 / 41)$ \\
Pulsatile pattern & $93.5(29 / 31)$ & $100(37 / 37)$ & $100(29 / 29)$ & $94.9(37 / 39)$ \\
\hline
\end{tabular}

treated successfully with conservative medical treatment. In 37 infants the ductus was not clinically significant and closed spontaneously.

The mean (SD) gestational ages were: 26.9 (2.9) weeks (range 23.2 to 34.2 weeks) and 29.2 (2.5) weeks (range 23.6 to 34.2 weeks); the mean birthweights were 857 (278)g (range 495 to $1485 \mathrm{~g}$ ) and 1132 (260)g (range 584 to $1470 \mathrm{~g}$ ) for the clinically significant PDA and non-clinically significant PDA groups, respectively. Artificial surfactant was administered to 26 infants $(83.9 \%)$ and 27 infants $(73.0 \%)$ in the clinically significant PDA and nonclinically significant PDA groups, respectively. Infants with clinically significant PDA significantly were of lower gestational age $(P<0.001)$ and birthweight $(\mathrm{P}=0.001)$, but there was no significant difference in amount of surfactant supplement given $(P=0.28)$ to both groups. The mean age at which indomethacin treatment was started $1.9(0.9)$ days (range 0.2 to 4 days).

The longitudinal echocardiographic change in shunt flow pattern according to age in days for both groups is summarised in table 1 . The pulmonary hypertension pattern was a common finding in early postneonatal life. The first instance of the pulsatile pattern was mostly recorded between days 2 and 3 of life. The sequence of transition for infants with clinically significant PDA was from the pulmonary hypertension pattern to the growing pattern to the pulsatile pattern to the closing pattern to the closed pattern. For non-clinically significant PDA, the pattern changed from pulmonary hypertension, to closing, to closed. In seven infants the growing pattern was recorded only once. The clinical status of PDA in each infant when various Doppler PDA patterns were first recorded up to 7 days of life is summarised in table 2 . The pulsatile pattern was recorded only in infants with clinically significant PDA or in those in whom this subsequently developed.

When indomethacin was started the flow patterns were as follows: growing pattern $(n=4)$, pulsatile pattern $(n=25)$. In two infants the ductus reopened at day 17 when both had a clinically significant PDA with a pulsatile pattern, in another infant the ductus reopened on day 21 when there was no clinically significant PDA and a closing pattern. These results show that the most discriminant Doppler pattern for infants with clinically significant PDA requiring indomethacin therapy was the pulsatile pattern. Using the shunt flow patterns to predict the risk of developing clinically significant PDA, the first growing pattern recorded gave a sensitivity of $64.5 \%$ and specificity of $81.1 \%$, and the first pulsatile pattern recorded had a sensitivity of $93.5 \%$ and specificity of $100 \%$ (table 3 ).

\section{Discussion}

Premature infants with clinically significant PDA are at increased risk of adverse outcomes. Left to right shunting through a PDA is associated with greater severity of respiratory distress syndrome and requires more ventilatory treatment, which increases the risk of chronic lung disease. ${ }^{19}$ PDA also adversely affects blood pressure, ${ }^{20}$ the systemic perfusion patterns to many organ systems, ${ }^{21}$ and is associated with an increased risk of intraventricular haemorrhage or ischaemic cerebral damage ${ }^{22}{ }^{23}$ and necrotising enterocolitis. ${ }^{24}$ The effects of surfactant replacement on pulmonary vascular resistance lead to the clinical emergence of PDA earlier and more frequently in preterm infants. ${ }^{1-3}$ All of these prompted early treatment with prophylactic indomethacin to improve outcomes. A recent review on prophylactic indomethacin ${ }^{25}$ reveals that there is a trend towards reduced neonatal mortality, a significantly reduced incidence of symptomatic PDA, and grade 3 and 4 intraventricular haemorrhage in treated infants. But there is no evidence that prophylactic indomethacin treatment affects respiratory outcomes and more data are needed on the long term neurodevelopemental outcomes and possible adverse effects, especially the incidence of necrotising enterocolitis and clinically important episodes of impaired renal function. Another recent meta-analysis of different treatment strategies ${ }^{26}$ has found that early treatment of a clinically significant PDA with indomethacin significantly reduces bronchopulmonary dysplasia, the duration of mechanical ventilation, and necrotising enterocolitis, but prophylactic indomethacin does not offer any additional advantage in these aspects compared with early treatment. A major concern with prophylactic indomethacin treatment is that only some 
infants will actually develop a clinically significant PDA and/or intraventricular haemorrhage, and it is only these infants that would benefit from the prophylaxis, but all infants would be exposed to the potential adverse effects of treatment. Earlier and more accurate prediction of the development of a clinically significant PDA would therefore permit appropriate preventive interventions and avoid inappropriate treatment and the ensuing adverse effects.

Clinically significant PDA is difficult to diagnose accurately in early postnatal life. ${ }^{4-7}$ Therefore, echocardiography has become essential in the evaluation of clinically significant ductal shunting. ${ }^{8-17}$ Most studies of echocardiographically derived indices correlate their findings with clinically significant PDA. We believe that what is most important is whether the echocardiographically derived index can detect prospectively the development of clinically significant PDA.

Our study has shown that four patterns of PDA shunt flow can be identified using pulsed Doppler echocardiography, and the longitudinal observation of the change in Doppler pattern can provide an understanding of the haemodynamics of ductal shunting and is useful for the prediction of risk of clinically significant PDA. The pulmonary hypertension pattern is a common finding in early postnatal life (tables 1 and 2), which is the reflection of a bi-directional shunt with only a little left to right shunting in the presence of a high pulmonary artery resistance. Therefore, it is not a good predictor of the risk of developing clinically significant PDA. In non-clinically significant PDA, accompanied by a fall in pulmonary artery resistance, the left to right shunting through a small enough ductus produces a very rapid velocity characterised by the closing pattern. In clinically significant PDA, the pulmonary hypertension, growing, and pulsatile patterns represent a continuum and are evidence of falling pulmonary artery resistance in the presence of a ductus which is not completely constricted and becomes haemodynamically clinically significant. Of 31 infants with clinically significant PDA, 29 infants were treated with indomethacin. The flow patterns at which indomethacin treatment was started were: growing pattern $(n=4)$ and pulsatile pattern $(n=25)$. After indomethacin treatment most of the Doppler pattern became the closed pattern or closing pattern and then closed pattern accompanied by the disappearance of clinical signs of PDA. All the closing and closed patterns had non-clinically significant PDA, indicating spontaneous or pharmacological closure of the duct (table 2).

The growing pattern was more frequently documented in infants with clinically significant PDA than in those with non-clinically significant PDA $(P=0.0001)$ (table 2). And among the 20 infants with clinically significant PDA in whom the growing pattern was documented, four infants had clinically significant PDA. After indomethacin treatment was started, two became the closing pattern and two became the pulsatile pattern; 16 infants subsequently became the pulsatile pattern and developed clinically significant PDA. This result shows that many PDA which subsequently become symptomatic were haemodynamically significant while clinically silent in early postnatal life. This finding implies that the growing pattern is a predictor for risk of developing clinically significant PDA. Interestingly, the pulsatile pattern was never recorded in infants with non-clinically significant PDA. This may reflect the fact that the infants with non-clinically significant PDA went through a brief transition of pulsatile pattern before the closing pattern developed and this phase may have beeen too short for us to recode it, or that the pulsatile pattern appeared exclusively in the process of development of a clinically significant PDA shunt. Of the infants in whom the pulsatile pattern was first recorded, $69 \%$ (20/29) had clinically significant PDA and 31\% (9/29) subsequently developed clinically significant PDA (table 2). According to our longitudinal study of Doppler PDA flow pattern, a growing or pulsatile pattern indicates a continuing risk of developing clinically significant PDA. Using the first documented growing pattern to predict the risk of developing clinically significant PDA, we were able to predict a clinically significant PDA with $64.5 \%$ sensitivity and $81.1 \%$ specificity; the first documented pulsatile pattern gave a $93.5 \%$ sensitivity and $100 \%$ specificity during the first four days of life in infants weighing less than $1500 \mathrm{~g}$ and undergoing mechanical ventilation (table 3 ). If all the infants in our study had been given prophylactic indomethacin, $55 \%(37 / 68)$ of the infants would have been treated unnecessarily. Using the first recording of the growing or pulsatile pattern during the first four days of age as an indicator for targeting treatment, would have resulted in appropriate treatment for $100 \%$ of infants who eventually developed clinically significant PDA. Only $10 \%(7 / 68)$ of all the infants would have been treated unnecessarily with indomethacin. These $10 \%$ of infants who had a growing pattern PDA shunt with the potential to progress to clinically significant PDA may also have benefited from pharmacological closure, even though the ductus eventually would have closed spontaneously.

In conclusion, the longitudinal echocardiographic assessment of PDA shunt flow pattern can exactly reflect the haemodynamic changes of the PDA. It is a simple technique to learn and is more convenient than calculating the PDA shunting volume from both ventricular outputs to predict a haemodynamically clinically significant PDA. The pulmonary hypotension pattern indicates high pulmonary artery resistance with little left to right shunt. The closed pattern indicates a closed ductus that is also incompatible with a left to right shunt. The closing pattern suggests a restrictive PDA small enough to prevent a clinically significant left to right shunt. The growing and pulsatile patterns reflect falling pulmonary artery resistance in the presence of a large ductus with clinically significant left to right shunt, and are an indicator for risk of developing 
clinically significant PDA. Clinicians need to recognise that once pulmonary artery resistance has fallen, if the closing pattern has not developed, the ductus is large enough to create problems. We recommend a daily simple screening echocardiogram during the first 4 days of life with an examination of the PDA Doppler pattern for premature infants at high risk of developing clinically significant PDA. At that stage, the closing or closed pattern indicates infants not at risk of developing clinically significant PDA, whereas the growing or pulsatile pattern indicates a continuing risk of developing clinically significant PDA.

This study was supported in part by a grant from the Tokyo Metropolitan Department of Health.

1 Kaapa P, Seppanen M, Kero P, Saraste M. Pulmonary hemodynamics after synthetic surfactant replacement in neonatal respiratory disease syndrome. I Pediatr 1993;123:115-9.

2 Reller MD, Buffkin DC, Colasurdo MA, Rice MT, McDonald RW. Ductal patency in neonates with respiratory
disease syndrome: a randomised surfactant trial. Am $\mathcal{F}$ Dis disease syndrome: a rand

3 Reller MD, Rice MT, McDonald RW. Review of studies evaluating ductal patency in the preterm infant. $\mathcal{F}$ Pediatr 1993;122:S59-S62.

4 Duddel GG, Gersony WM. Patent ductus arteriosus in neonates with severe respiratory disease. $f$ Pediatr 1984;104:915-20.

5 Mellander M, Larsson LE, Ekstrom-Jodal B, Sabel KG. Prediction of symptomatic patent ductus arteriosus in preterm infants using Doppler and M-mode echocardiography. Acta Paediatr Scand 1987;76:553-9.

6 Skelton R, Evans NJ, Smythe J. A blinded comparison of clinical and echocardiographic evaluation of the preterm infant for patent ductus arteriosus. $\mathcal{F}$ Pediatr Child Health 1993;29:A61.

7 Evans NJ, Iyer P. Change in blood pressure after treatment of patent ductus arteriosus with indomethacine. Arch Dis of patent ductus arter

8 Silverman NH, Levis AB, Heymann MA, Rudoph AM. Echocardiographic assessment of ductus arteriosus shunt in preterm infants. Circulation 1974:50:821-5.

9 Huhta JC, Cohen M, Gutgesell HP. Patency of the ductus arteriosus in normal neonates: two dimensional echocardiography vs Doppler assessment. $\mathcal{F} \mathrm{Am}$ Coll Cardio 1984;4:561-4.
10 Huhta JC, Gutgesell HP, Latson LA, Huffines FD. Two-dimensional echocardiographic assessment of the Two-dimensional echocardiographic assessment of the aorta in infants and children
Circulation 1984;70:417-24.

11 Gentile R. Stevenson GM, Dooley T. Frankline D, Kawabori I, Pearlman A. Pulsed Doppler echocardiographic determination of time of ductal closure in normal newborn infants. F Pediatr 1981;98:443-8.

12 Smallhorn JF, Gow R. Olley PM, et al. Combined noninvasive assessment of the patent ductus arteriosus in the preterm infants before and after indomethacine treatment. Am $\mathcal{F}$ Cardiol 1984;54:1300-4.

13 Ramsey JM, Murphy DJ, V'ck GW, Courtney JT, GarciaPrats JA, Huhta JC. Response of the patent ductus arteriosus to indomethacine treatment. $A m$ f Dis Child 1987;141:294-7.

14 Wilson N. Dickison DF, Goldberg SJ, Scott O. Pulmonary artery velocity patterns in ductus arteriosus. Br Heart 7 1984;52:462-4.

15 Shiraishi $\mathrm{H}$. Yanagisawa $M$. Bidirectional flow through the ductus arteriosus in normal newborn: evaluation by Doppler color flow imaging. Pediatr Cardiol 1991;12:201-5.

16 Houston AB, Gnanapragasam JP, Lim MK, Doig WB, Coleman EN. Doppler ultrasound and the silent ductus arteriosus. Br Heart F 1991;65:97-9.

17 Kluckow M, Evans N. Early echocardiographic prediction of symptomatic patent ductus arteriosus in preterm infants undergoing mechanical ventilation. $\mathcal{f}$ Pediat 1995;127:774-9.

18 Miyama N, Itoh R, Ohmori I, Su BH, Shimizu M, Watanabe $\mathrm{T}$. Intravenous indomethacin treatment in very low birthweight infants with PDA: a retrospective study of 95 infants. Acta Neonat fpn 1995;31:854A. (in Japanese)

19 Brown ER. Increased risk of bronchopulmonary dysplasia in infants with patent ductus arteriosus. $f$ Pediatr infants with $1979 ; 95: 865-6$.

20 Evans N, Moorcraft J. Effect of patency of the ductus arteriosus on pressure in very preterm infants. Arch Dis Child 1992;67:1169-73.

21 Shimada S, Kasai T, Konishi M, Fujiwara T. Effect of patent ductus arteriosus on left ventricular output and organ blood flow in preterm infants with respiratory distress syndrome treated with surfactant. F Pediatr 1994;125:270-7.

22 Lipman B, Server GA, Brazy JE. Abnormal cerebral hemodynamics in preterm infants with patent ductus arteriosus. Pediatrics 1982;69:778-81.

23 Martin CG, Snider AR, Katz SM, Peabody JL, Brady JP. Abnormal cerebral blood flow patterns in preterm infants with a large patent ductus arteriosus. $\mathcal{f}$ Pediatr 1982;101:587-93.

24 Ryder RW, Shelton JD, Guinan ME. Necrotizing enterocolitis: A prospective multicenter investigation. Am $\mathcal{F}$ Epidemiol 1980;112:113-23.

25 Fowlie PW. Prophylactic indomethacin: systemic review and meta-analysis. Arch Dis Child 1996;74:F8 1-7.

26 Clyman RI. Recommendation for the postnatal use of indomethacin: An analysis of four separate treatment strategies. F Pediatr 1996;128:601-7. 